\title{
Genomic characterization and phylogenetic analysis of the first SARS-CoV-2 variants introduced in Lebanon
}

\author{
Rita Feghali ${ }^{\text {Equal first author, } 1}{ }^{1}$, Georgi Merhi ${ }^{\text {Equal first author, } 2}{ }^{,}$Aurelia Kwasiborski ${ }^{3}$, Veronique Hourdel ${ }^{3}$, Nada Ghosn ${ }^{4}$, Sima \\ Tokajian ${ }^{\text {Corresp. } 2}$ \\ 1 Department of Laboratory Medicine, Rafik Hariri University Hospital, Beirut, Lebanon \\ 2 Department of Natural Sciences, Lebanese American University, Byblos, Lebanon \\ 3 Laboratory: Environment and Infectious Risks, Pasteur Institute, Paris, France \\ 4 Epidemiological Surveillance Unit, Ministry of Public Health, Beirut, Lebanon \\ Corresponding Author: Sima Tokajian \\ Email address: stokajian@lau.edu.lb
}

Background. In December 2019, the COVID-19 pandemic initially erupted from a cluster of pneumonia cases of unknown origin in the city of Wuhan, China. Presently, it has almost reached 94 million cases worldwide. Lebanon on the brink of economic collapse and its healthcare system thrown into turmoil, has previously managed to cope with the initial SARS-CoV-2 wave. In this study, we sequenced 11 viral genomes from positive cases isolated between February $2^{\text {nd }} 2020$ and March $15^{\text {th }} 2020$. Methods. Sequencing data was quality controlled, consensus sequences generated, and a maximum-likelihood tree was generated with IQTREE v2. Genetic lineages were assigned with Pangolin v1.1.14 and single nucleotide variants (SNVs) were called from read files and manually curated from consensus sequence alignment through JalView v2.11 and the genomic mutational interference with molecular diagnostic tools was assessed with the CoV-GLUE pipeline. Phylogenetic analysis of whole genome sequences confirmed a multiple introduction scenario due to international travel. Results. Three major lineages were identified to be circulating in Lebanon in the studied period. The B.1 (20A clade) was the most prominent, followed by the B.4 lineage (19A clade) and the B.1.1 lineage (20B clade). SNV analysis showed 15 novel mutations from which only one was observed in the spike region. 


\section{Genomic Characterization and Phylogenetic Analysis of the 2 First SARS-CoV-2 Variants Introduced in Lebanon}

3

4

$5{ }^{\wedge}$ Rita A. Feghali ${ }^{1}$, Georgi S. Merhi²^, Aurélia Kwasiborski ${ }^{3}$, Véronique Hourdel ${ }^{3}$, Nada O.

6 Ghosn $^{4}$ and ${ }^{\wedge}$ Sima T. Tokajian ${ }^{2 *}$

$7 \quad{ }^{1}$ Departement of Laboratory Medicine, Rafik Hariri University Hospital, Beirut, Lebanon

$8 \quad{ }^{2}$ Department of Natural Sciences, Lebanese American University, Byblos, Lebanon.

9 ' ${ }^{3}$ Laboratory: Environment and Infectious Risks, Pasteur Institute, Paris, France.

$10{ }^{4}$ Epidemiological surveillance unit, Ministry of Public Health, Beirut, Lebanon.

11

12

13

14 Corresponding Author:

15 Sima Tokajian, $\mathrm{PhD}$,

16 Lebanese American University,

17 P.O. Box 36 Byblos, Lebanon.

18

Fax: +961-9-944851, Phone: +961-9-547254 Ext: 2861.

19

Email address: stokajian@lau.edu.lb

20

21

22 
23 Abstract

24

Background. In December 2019, the COVID-19 pandemic initially erupted from a cluster of

pneumonia cases of unknown origin in the city of Wuhan, China. Presently, it has almost reached 94 million cases worldwide. Lebanon on the brink of economic collapse and its healthcare system thrown into turmoil, has previously managed to cope with the initial SARS-CoV-2 wave. In this study, we sequenced 11 viral genomes from positive cases isolated between February $2^{\text {nd }}$ 2020 and March $15^{\text {th }} 2020$.

31 Methods. Sequencing data was quality controlled, consensus sequences generated, and a maximum-likelihood tree was generated with IQTREE v2. Genetic lineages were assigned with Pangolin v1.1.14 and single nucleotide variants (SNVs) were called from read files and manually curated from consensus sequence alignment through JalView v2.11 and the genomic mutational interference with molecular diagnostic tools was assessed with the CoV-GLUE pipeline. Phylogenetic analysis of whole-genome sequences confirmed a multiple introduction scenario

37 due to international travel.

Results. Three major lineages were identified to be circulating in Lebanon in the studied period. The B.1 (20A clade) was the most prominent, followed by the B.4 lineage (19A clade) and the B.1.1 lineage (20B clade). SNV analysis showed 15 novel mutations from which only one was observed in the spike region.

In December 2019, unknown cases of pneumonia were detected in the city of Wuhan, Hubei province, China. Infected individuals exhibited symptoms similar to that of severe acute

47 respiratory syndrome (SARS) (Li et al., 2020). Deep sequencing identified the causative agent as 
48 a novel $\beta$-coronavirus, named nCoV-2019, later, renamed as SARS-CoV-2 (Gralinski \&

49 Menachery, 2020). Since then, the World Health Organization (WHO) has classified the spread

50 of the virus as a global pandemic (Astuti \& Ysrafil, 2020) and as of January 18, 2021, there was

51 a total of 93,194,922 confirmed cases with 2,014,729 deaths (https://covid19.who.int/). COVID-

52 19's common clinical symptoms include, but not limited to, fever, development of a dry cough,

53 myalgia/fatigue, dyspnea, headaches and pneumonia (Zhou et al., 2020; Huang et al., 2020).

54 Molecular characterization of the SARS-CoV-2 genome showed $79.6 \%$ sequence similarity to

55 SARS-CoV and 96\% to the RaTG13 bat-CoV (Zhou et al., 2020) supporting that bats

56 (Rhinolophus affinis) may have acted as a reservoir for the SARS-CoV-2 progenitor (Andersen

57 et al., 2020). Interestingly, analysis of SARS-CoV-2's structural protein ORF3a revealed highly

58 conserved protein domains within homologs from civet, pangolin and SARS-like bat-CoVs (Issa

59 et al., 2020).

60 Despite the difficult economic situation and the waning infrastructure of its healthcare sector,

61 Lebanon has successfully managed the initial COVID-19 wave. As of 21 February, 2020, there

62 has been 249,158 confirmed cases with 1,866 total deaths, with $1.4 \%$ of cases being attributed to

63 exposure outside Lebanon and $98.6 \%$ due to local community spread of the SARS-CoV-2 virus

64 (https://www.moph.gov.lb/en/Pages/2/193/esu\#/en/Pages/2/24870/novel-coronavirus-2019-).

65 In this study, we performed a comprehensive genomic analysis of 11 SARS-CoV-2 isolates

66 recovered from Lebanese individuals during the first phase of the pandemic. We also looked at

67 their phylogeny based on location and date of exposure. Finally, we determined the single

68 nucleotide variants (SNVs) and amino acid changes and compared our results with worldwide

69 disseminating SARS-CoV-2 variants to assess epidemiological relatedness.

70 


\section{Materials \& Methods}

73

74

75

76

77

78

79

80

81

82

83

84

85

86

87

88

89

90

91

92

93

94

95

\section{COVID-19 response in Lebanon and genomic sequencing}

The 11 cases (Table 1) undertaken in this study were detected and then selected randomly

between 21-February-2020 and 15-March-2020 and designated as S1-S11. The clinical data were collected as part of the quarantine monitoring measures at the Rafik al Hariri University Hospital (RHUH) with the support of the Lebanese Ministry of Public Health (MoPH). Required written informed consent was not obtained for the first studied cases due to the pressing need for data collection in the early stages of the outbreak. This study was approved by the Institutional Review Board (IRB) of the Lebanese American University (IRB \#: AU.SAS.ST2.19/May/2020). RNA was extracted from the specimens using the Qiagen QIAamp Viral RNA Mini kit (QIAGEN, Hilden, Germany) by following the manufacturer's instructions. An qRT-PCR corresponding to the Charité protocol (published on Jan 17, 2020) was used for detection of SARS-CoV-2. The assay relies on a first-line E gene screening, followed by a confirmatory assay using the RNA dependent RNA polymerase $(R d R p)$ gene and a synthetic RNA positive control (Charité virology institute - Universitätsmedizin Berlin, Berlin, Germany). A $25 \mu 1$ reaction was set up containing $1 \mu 1$ of forward primer $(10 \mu \mathrm{M}), 1 \mu 1$ of reverse primer $(10 \mu \mathrm{M})$, $0.5 \mu \mathrm{l}$ of probe $(10 \mu \mathrm{M}), 5 \mu \mathrm{l}(100 \mathrm{ng} / \mu \mathrm{l})$ of extracted RNA, $12.5 \mu \mathrm{l}$ of $2 \mathrm{X}$ reaction buffer and $1 \mu \mathrm{l}$ of reverse Transcriptase/Taq Polymerase mixture (Invitrogen, NY, USA) provided with the Superscript III One step RT-PCR system (Corman et al., 2020).

Thermal cycling was performed at $55^{\circ} \mathrm{C}$ for $10 \mathrm{~min}$ for reverse transcription, followed by $95^{\circ} \mathrm{C}$ for $3 \mathrm{~min}$ and then $45 \mathrm{cycles}$ of $95^{\circ} \mathrm{C}$ for $15 \mathrm{sec}$ and $58^{\circ} \mathrm{C}$ for $30 \mathrm{sec}$. cDNA amplification was done through the tiled amplification approach and following ARCTIC's network recommended protocol (Quick, 2020). 
96 Sequencing libraries were prepared from tiled amplicons using the Miseq reagent kit v3

97 (Illumina, CA, USA) and sequenced on an Illumina MiSeq system using $250 \mathrm{bp}$ paired-end reads

98 and following the manufacturer's instructions. Sequences were randomly labeled from S1 to S11

99 based on their loading order onto the sequencer.

\section{Consensus sequences}

101 Raw sequencing reads were input into FastQC v0.11.9

102 (https://www.bioinformatics.babraham.ac.uk/projects/fastqc/) for quality assessment and

103 subsequent quality control was performed through FQCleaner v3.0 (Criscuolo \& Brisse, 2013)

104 with a 28 quality score threshold and a minimum read length of 30. Quantified reads were

105 mapped to the Wuhan-Hu-1 reference genome (MN908947.3) using BWA v0.7.17 with the

106 'MEM`alignment algorithm (https://arxiv.org/abs/1303.3997). Consensus sequences were

107 generated for all 11 SARS-CoV-2 isolates using SAMtools v1.9 (http://www.htslib.org/) and

108 veftools v0.1.16 (https://vcftools.github.io/index.html).

\section{Phylogenetic analysis}

110 Six high quality $(\mathrm{N}<0.5 \%$; Length $>29,000$ bp) genomes (S1, S2, S4, S5, S8, S9) were selected

111 for phylogenetic analysis. Reference sequences $(n=55)$ were chosen based on several criteria

112 including phylogenetic placement of reference genomes, collection dates, history of exposure

113 (travel), GISAID's BLAST feature within the EpiCoV TM browser (https://www.epicov.org/epi3)

114 and overall genome quality/completeness to avoid sequence-based bias (Table S1).

115 Sequences were aligned with MAFFT v7.467 (Katoh \& Standley, 2013). The resulting alignment

116 was used in masking terminal regions and gaps with Nextstrain's custom Python script

117 (https://github.com/nextstrain/ncov/tree/master/scripts). The alignment was input into

118 ModelFinder to assess the best fit substitution model (Kalyaanamoorthy et al., 2017). A 
119 maximum likelihood (ML) tree was generated with IQ-TREE v2(Minh et al., 2020) using the 120 TIM2+F substitution model. Boot-strap support was established through 1000 iterations for 121 UFBoot (Ultra-Fast Bootstrapping) and SH-like approximate likelihood ratio test (SH-aLRT)

122 (Guindon et al., 2010). The consensus tree was visualized with the interactive tree of life v4 123 (IToL; https://itol.embl.de/)(Letunic \& Bork, 2019).

124 We assigned genetic lineages based on three commonly used systems including the recently 125 proposed dynamic classification using Pangolin tool v1.1.14 (https://github.com/hCoV-

126 2019/pangolin)(Rambaut et al., 2020), the Nextstrain classification and GISAID's internal 127 classification.

\section{Comparative genome and spike (S) protein analyses}

129 SAM files from read alignment for all samples were converted into BAM files with SAMtools 130 v1.9 (http://www.htslib.org/). Using bcftools v1.9 (https://samtools.github.io/bcftools/), variants 131 were called and extracted through the "mpileup" and "call" commands with ploidy set to 1 and 132 invoking the multiallelic-caller through the "-m" flag. Obtained variants were filtered with the 133 "varfilter" command using the custom perl script vcfutils.pl (http://www.htslib.org/).

134 Consensus genomes were aligned against Wuhan-Hu-1 (MN908947.3) with MAFFT 135 v7.467(Katoh \& Standley, 2013) and the resulting alignment was input in SNP-sites v2.5.1(Page 136 et al., 2016) to extract all identified SNPs (Table 2). The alignment was visualized (Fig. S1) with 137 JalView v2.11(Waterhouse et al., 2009) and polymorphic sites were manually curated (positions, 138 with low coverage, defined by $\mathrm{N}$ strings were omitted). For added stringency, sequences were 139 input into the CoV-GLUE analysis pipeline (http://cov-glue.cvr.gla.ac.uk/) where all SNPs and 140 amino acid variations were identified for all genomes. Potential interference with all available 141 diagnostic assays for SARS-CoV-2 was also investigated. 
142 Genome annotation was performed with Prokka v1.14.6(Seemann, 2014). Subsequently, spike

143 (S) protein amino acid sequences were extracted, aligned with MAFFT v.7467(Katoh \&

144 Standley, 2013) and visualized with JalView v2.11(Waterhouse et al., 2009).

145

146 Results

147

148 Clinical characteristics of patients

149 The first SARS-CoV-2 positive case was documented in Lebanon on 2/21/2020. By 3/15/2020, 150 Lebanon had a total of 108 positive cases (https://www.moph.gov.lb/maps/covid19.php). Among

151 the eleven patients, five were clinically asymptomatic $(\mathrm{n}=5)$ and four exhibited mild symptoms $152(\mathrm{n}=4)$. The remaining two patients displayed a severe form of COVID-19 (Fig 1). All patients 153 were hospitalized, including asymptomatic carriers as a form of quarantined isolation to slow 154 community spread (Fig 1). Four patients out of eleven $(n=4)$ were female and the median age 155 was 38.5 years and the range was 19 to 74 (Table 1 ).

156 Dates of symptom onset varied between the $17^{\text {th }}$ of February and $13^{\text {th }}$ of March (Fig 1). Travel 157 history differed between patients and included countries such as Iran, France, Italy, and the 158 United Kingdom (Table 1), which was consistent with multiple introduction incidences. The 159 initial signs and symptoms were headache $(n=2)$, rhinorrhea $(n=2)$ and flu-like symptoms $(n=2)$.

160 Over the course of illness, a patient reported abdominal pain and diarrhea while another only 161 suffered from dyspnea (Table 1). The two patients with severe COVID-19, initially developed

162 flu-like symptoms followed by severe dyspnea and acute respiratory distress syndrome (Fig 1). 163 Phylogenetic Analysis

164 The SARS-CoV-2 isolates recovered from Lebanon clustered, and according to Nextstrain's 165 classification, in three distinct clades namely: 19A (93\% bootstrap support), 20A (81\% bootstrap 
166 support) and 20B (98\% bootstrap support) (Fig 2). S5 (EPI_ISL_450512) and S9

167 (EPI_ISL_450515) were grouped closely to sequences from India (EPI_ISL_435106,

168 EPI_ISL_421667, EPI_ISL_435101) and Kuwait (EPI_ISL_416458) in clade19A. Interestingly,

169 S9 displayed less phylogenetic divergence than S5 based on the distance observed in the

170 phylogenetic tree and polymorphic sites differences. Both patients had different exposure

171 histories (Table 1).

172 S1 (EPI_ISL_450508), S2 (EPI_ISL_450509) and S8 (EPI_ISL_450514) clustered within clade

173 20A. S2 and S8 were closely related to one recovered from Egypt (EPI_ISL_430820). However,

174 S2 was phylogenetically more related to the isolate from Egypt than S8, with both being linked

175 to local community transfer (Fig 2, Table 1). S1 was recovered from a patient with travel history

176 to Egypt and clustered close to sequences recovered from Saudi Arabia (EPI_ISL_437697), USA

177 (EPI_ISL_447844) and Taiwan (EPI_ISL_444276) (Table 1). Additionally, S1 showed lesser

178 evolutionary distance than S2 and S8 (Fig 2) indicating less genomic diversity. S4

179 (EPI_ISL_450511) on the other hand, was of the same superclade as that of S1, S2 and S8, but

180 clustered under 20B (Fig 2). It also showed proximity to sequences recovered from Europe and

181 more so from England (EPI_ISL_448804), Belgium (EPI_ISL_417422) and Lithuania

182 (EPI_ISL_450496), which was in accordance with the patient's travel history.

183 We also assigned the lineages using pangolin v1.1.14, and the results (Table 3) obtained were 184 consistent (Fig 2). S5 and S9 were assigned to the B.4 lineage alongside S3 (Table 3). S4 and S6

185 were assigned to lineage B.1.1, while the remaining isolates clustered under the B.1 lineage 186 representing clade 20A (Fig 2). The GISAID nomenclature could be also correlated with the

187 lineage assignments. S5 and S9 were included in the O clade with all the others fitting under the 188 super G clade: S4 and S6: GR clade and S1, S2, S7, S8, S10 and S11: GH clade. 
189 SNVs in Sequenced genomes and the S protein.

190 The obtained consensus genomes of S3 and S6 showed low coverage values being $70.2 \%$ and $19192.6 \%$, respectively. Accordingly, polymorphism could not be confirmed and as such were 192 excluded from downstream analysis. Table 2 shows all detected SNP (high and low depth) sites 193 across the 11 aligned genomes. The genetic variation within the aligned genomes was relatively 194 low, with a minimum of 5 and a maximum of 19 SNPs (median: 11 SNPs). In S1, S2, S7, S8, 195 S10, and S11 three amino acid (AA) changes were detected within: ORF1ab within the non196 structural protein 12 (nsp12) P323L, spike (S) protein D614G, and ORF3a's Q57H (Table 2).

197 Only P323L and D614G AA changes were detected in S4 with two other additional mutations in 198 the N protein (R203K and G204R). S5 and S9 shared four common AA changes in nsp2 (R27C, 199 V198I), nsp4 (M33I) and nsp6 (L37F). Interestingly, S5 had an additional mutation in nsp2 200 (A360V) and a novel mutation in nps3 (I1683V), while S9 displayed an AA change in the spike 201 (S) protein at position 153 (M153I).

202 We also detected in S7, S8, S10 and S11 novel amino acid changes in multiple coding regions 203 within the SARS-CoV-2 genome (Table 3). Furthermore, S8 had a novel frame-shift (fs) deletion 204 at nucleotide position 8651 , leading to a change in nsp4 at position 33 and as a result replacing a 205 methionine residue by the aromatic residue phenylalanine (M33Ffs).

206 Analysis of the polymorphic sites in the context of diagnostics revealed multiple hot-spots where 207 accumulating nucleotide polymorphisms could interfere with the diagnostic schemes based on 208 the ARCTIC network amplicon sequencing primers. In particular, changes such as: C13381T, 209 G28881A, G28882A and G28883C in S2, S4, S6, S8 and S1 1 may interfere with the specificity 210 primers and probes designed by the Chinese Center for Disease Control and Prevention (China 
211 CDC) and used for the detection of 2019-nCoV through targeting the ORF1ab encoded

212 polymerase and $\mathrm{N}$ protein.

213 We also compared the S protein within the 11 genomes through an intra-isolate alignment. The

214 D614G amino acid change was detected in all the isolates except S3, S5 and S9. This mutation,

215 however, would not cause changes in the receptor binding domain (RBD-Spike:455-505) or in

216 the polybasic cleavage site unique (PCS-Spike: 681-686) to SARS-CoV-2. Finally, M153I and

217 M177R mutations were only observed in isolates S9 and S11, respectively.

218

\section{Discussion}

220

221 We aimed in this study at studying the SARS-CoV-2 isolates recovered at the early stages of the 222 COVID-19 outbreak in Lebanon. To that end, we sequenced eleven genomes, including patient 223 zero, collected from 21/02/2020 until 15/03/2020. We investigated the phylogenetic and

224 epidemiological relatedness of the genomes and found three lineages circulating since the start of 225 the local outbreak. Furthermore, several novel amino acid mutations in ORF1ab encoding for 226 non-structural proteins and other structural proteins were detected.

227 Our results showed that S3 and S5 belonged to the B.4 lineage (O clade on GISAID) with a 228 travel history linked to Iran (Table 1). This is consistent with previous reports where distinct 229 clades were attributed to returnees from Iran, with all the genomes clustering under the B.4 230 lineage (O clade) (Eden et al., 2020; Potdar et al., 2020). Moreover, sequences that clustered 231 close to S5 in Fig 2 were also associated with possible exposure and travel history to Iran. It is 232 noteworthy, that the first COVID-19 case in Iran was officially reported on February $19^{\text {th }}$ 233 (https://covid19.who.int/region/emro/country/ir) whereas the first case (S7) in Lebanon was on 234 February $21^{\text {st }}$ and linked to a patient returning from the city of Qom, Iran 


\section{5 (https://www.moph.gov.lb/en/Media/view/27426/coronavirus-disease-health-strategic-}

236 preparedness-and-response-plan-), and which clustered under the B.1 lineage (GH clade).

237 S4 and S9 were however, both linked to patients with travel history to the United Kingdom

238 (UK), but clustered in two different lineages, B.1.1 and B.4, respectively. Detailed analysis of

239 the European sub-clusters by Nextstrain (Hadfield et al., 2018) revealed that the UK outbreak

240 was largely rooted in B.1 and B.1.1 lineages which was in agreement with the analysis from

241 GISAID's EpiCoV ${ }^{\mathrm{TM}}$ database (Shu \& McCauley, 2017). The outbreak in Europe was mainly

242 linked to isolates clustering under clade $\mathrm{G}$ and its variants GH/GR. Available data suggested that

243 sequences linked to travel history to the UK represented the early stages of the outbreak with the

244 B.4 lineage being actively circulating at the time.

245 The predominant lineage in this study was B.1 (clade GH) (S1, S2, S7, S8, S10, S11). Figure 2

246 shows that S2 and S8 were closely related to SARS-CoV-2 recovered in Egypt

247 (EPI_ISL_430820) and somewhat distant from S1. S1 was linked to travel history to Egypt,

248 while S2 and S8 to local community spread suggesting potential multiple introductions

249 especially with the observed discrepancies in the number of SNPs in S8 compared to S1 (Table

$2503)$.

251 S protein amino acid changes revealed three variants among the sequenced isolates. Previously,

252 Bhattacharyya et al. (2020) (Bhattacharyya et al., 2020) suggested the widespread dominance of 253 SARS-CoV-2 with D614G/A23403G substitution in Europe and North America (Table 2). The

254 delC allele in TMPRSS2, common in Europe and North America, facilitated the entry of the 255 614G subtype into host cells, thus accelerating the spread of 614G subtype (Bhattacharyya et al., 256 2020). 
258 A novel S protein mutation (M177R) was detected in the S1 subunit in one of the isolates in this 259 study (S11) (Table 3), but at this point it is not clear whether it has any implications on its 260 interaction with the ACE2 receptor. Additionally, the $R d R p$ mutation P323L (C14408T), 261 generally detected in isolates recovered from Europe and North America (Pachetti et al., 2020), 262 was also detected in the sequenced isolates from Lebanon, and was consistent with the metadata 263 further showing multiple introduction points from Europe (UK, Italy, France). Mutations in the $264 R d R p$ are of interest with the encoded polymerase being an important target for current 265 therapeutic polymerase inhibitors (Pachetti et al., 2020), and with our data revealing several 266 novel mutations in nsp12 (Table 3).

\section{Conclusions}

268

269 The estimated mutation rate driving the genomic global diversity of the virus has been 270 determined at approximately $6 \times 10^{-4}$ nucleotides/genome/year (van Dorp et al., 2020). The 271 accumulated genetic diversity in the form of SNVs in the SARS-CoV-2 genomes throughout the 272 COVID-19 pandemic serves as a tool to assess and quantify the genomic diversity, evolutionary 273 distribution, and epidemiological linkage of the virus (Yang et al., 2020). With daily confirmed 274 cases on an exponential rise in Lebanon

275 (https://www.moph.gov.lb/en/Pages/2/193/esu\#/en/Pages/2/24870/novel-coronavirus-2019-) and 276 the rapid emergence of novel variants of concern (Volz et al., 2021), further sequencing efforts 277 are urgently needed to assess the spread and phylogenomic characteristics of SARS-CoV-2 in 278 Lebanon and keep track of the emerging variants which is much needed to mitigate the spread, 279 and for vaccine development and efficacy. This study offers a comprehensive genomic snapshot 280 of the earlier stages of the local outbreak. Importantly, our analysis highlights the viral lineages 
281 and genomic mutations identified as the root of the Lebanese outbreak which are also reflective

282 of the situation in neighboring regions that lack genomic and epidemiological data.

\section{Acknowledgements}

284

285

286

287

288

289

290

291

292

293

294

295

296

297

298

299

300

301

302

303

We thankfully acknowledge the personnel and laboratories who have generated and submitted sequences to the GISAID's EpiCoV'TM database. This study does not declare ownership of these sequences. The openly available data was used to compare our results within an international framework and to provide further information about the phylogenomic status and the spreadability of the SARS-CoV-2 in countries with little to non-existing epidemiological and genomic data. We also acknowledge Dr Guillain Mikaty, Dr Valerie Caro and Dr Jean-Claude Manuguerra from Institut Pasteur, for their technical assistance and support in the sequencing of the SARS-CoV-2 samples.

The authors declare that there is no existing conflict of interests.

\section{Funding:}

The sequencing of the SARS-CoV-2 samples was financed by the MediLabSecure project, founded by the European Commission (DEVCO: IFS/2018/402-247). This study was also financed by the Strategic Research Review Committee (Grant \# SRRC-R-2019-38) at the Lebanese American University and by the National Council for Scientific Research (Grant \#00993).

References

Andersen KG, Rambaut A, Lipkin WI, Holmes EC, Garry RF. 2020. The proximal origin of SARS-CoV-2. Nature Medicine:1-3. DOI: 10.1038/s41591-020-0820-9. 
304 Astuti I, Ysrafil. 2020. Severe Acute Respiratory Syndrome Coronavirus 2 (SARS-CoV-2): An 305 overview of viral structure and host response. Diabetes \& Metabolic Syndrome. DOI: 306

307 308 309 310 10.1016/j.dsx.2020.04.020.

Bhattacharyya C, Das C, Ghosh A, Singh AK, Mukherjee S, Majumder PP, Basu A, Biswas NK. 2020. Global Spread of SARS-CoV-2 Subtype with Spike Protein Mutation D614G is Shaped by Human Genomic Variations that Regulate Expression of TMPRSS2 and MX1 Genes. bioRxiv:2020.05.04.075911. DOI: 10.1101/2020.05.04.075911.

Corman VM, Landt O, Kaiser M, Molenkamp R, Meijer A, Chu DK, Bleicker T, Brünink S, Schneider J, Schmidt ML, Mulders DG, Haagmans BL, van der Veer B, van den Brink S, Wijsman L, Goderski G, Romette J-L, Ellis J, Zambon M, Peiris M, Goossens H, Reusken C, Koopmans MP, Drosten C. 2020. Detection of 2019 novel coronavirus (2019-nCoV) by real-time RT-PCR. Eurosurveillance 25. DOI: 10.2807/15607917.ES.2020.25.3.2000045.

Criscuolo A, Brisse S. 2013. AlienTrimmer: a tool to quickly and accurately trim off multiple short contaminant sequences from high-throughput sequencing reads. Genomics 102:500-506. DOI: 10.1016/j.ygeno.2013.07.011.

van Dorp L, Acman M, Richard D, Shaw LP, Ford CE, Ormond L, Owen CJ, Pang J, Tan CCS, Boshier FAT, Ortiz AT, Balloux F. 2020. Emergence of genomic diversity and recurrent mutations in SARS-CoV-2. Infection, Genetics and Evolution 83:104351. DOI: 10.1016/j.meegid.2020.104351.

Eden J-S, Rockett R, Carter I, Rahman H, de Ligt J, Hadfield J, Storey M, Ren X, Tulloch R, Basile K, Wells J, Byun R, Gilroy N, O’Sullivan MV, Sintchenko V, Chen SC, Maddocks S, Sorrell TC, Holmes EC, Dwyer DE, Kok J, Donovan L, Kumar S, Tran T, 
327

328

329

330

331

332

333

334

335

336

337

338

339

340

341

342

343

344

345

346

347

348

349

Ko D, Ngo C, Sivaruban T, Timms V, Lam C, Gall M, Gray K-A, Sadsad R, Arnott A. 2020. An emergent clade of SARS-CoV-2 linked to returned travellers from Iran. Virus Evolution 6. DOI: 10.1093/ve/veaa027.

Gralinski LE, Menachery VD. 2020. Return of the Coronavirus: 2019-nCoV. Viruses 12:135. DOI: $10.3390 / \mathrm{v} 12020135$.

Guindon S, Dufayard J-F, Lefort V, Anisimova M, Hordijk W, Gascuel O. 2010. New algorithms and methods to estimate maximum-likelihood phylogenies: assessing the performance of PhyML 3.0. Systematic Biology 59:307-321. DOI:

10.1093/sysbio/syq010.

Hadfield J, Megill C, Bell SM, Huddleston J, Potter B, Callender C, Sagulenko P, Bedford T, Neher RA. 2018. Nextstrain: real-time tracking of pathogen evolution. Bioinformatics (Oxford, England) 34:4121-4123. DOI: 10.1093/bioinformatics/bty407.

Huang C, Wang Y, Li X, Ren L, Zhao J, Hu Y, Zhang L, Fan G, Xu J, Gu X, Cheng Z, Yu T, Xia J, Wei Y, Wu W, Xie X, Yin W, Li H, Liu M, Xiao Y, Gao H, Guo L, Xie J, Wang G, Jiang R, Gao Z, Jin Q, Wang J, Cao B. 2020. Clinical features of patients infected with 2019 novel coronavirus in Wuhan, China. The Lancet 395:497-506. DOI:

$10.1016 / \mathrm{S} 0140-6736(20) 30183-5$.

Issa E, Merhi G, Panossian B, Salloum T, Tokajian S. 2020. SARS-CoV-2 and ORF3a: Nonsynonymous Mutations, Functional Domains, and Viral Pathogenesis. mSystems 5. DOI: $10.1128 / \mathrm{mSystems.00266-20.}$

Kalyaanamoorthy S, Minh BQ, Wong TKF, von Haeseler A, Jermiin LS. 2017. ModelFinder: fast model selection for accurate phylogenetic estimates. Nature Methods 14:587-589. DOI: $10.1038 /$ nmeth.4285. 
350 Katoh K, Standley DM. 2013. MAFFT Multiple Sequence Alignment Software Version 7:

351 Improvements in Performance and Usability. Molecular Biology and Evolution 30:772-

352 780. DOI: $10.1093 / \mathrm{molbev} / \mathrm{mst} 010$.

353 Letunic I, Bork P. 2019. Interactive Tree Of Life (iTOL) v4: recent updates and new

354 developments. Nucleic Acids Research 47:W256-W259. DOI: 10.1093/nar/gkz239.

Li Q, Guan X, Wu P, Wang X, Zhou L, Tong Y, Ren R, Leung KSM, Lau EHY, Wong JY, Xing X, Xiang N, Wu Y, Li C, Chen Q, Li D, Liu T, Zhao J, Liu M, Tu W, Chen C, Jin L, Yang R, Wang Q, Zhou S, Wang R, Liu H, Luo Y, Liu Y, Shao G, Li H, Tao Z, Yang Y, Deng Z, Liu B, Ma Z, Zhang Y, Shi G, Lam TTY, Wu JT, Gao GF, Cowling BJ, Yang B, Leung GM, Feng Z. 2020. Early Transmission Dynamics in Wuhan, China, of Novel Coronavirus-Infected Pneumonia. New England Journal of Medicine. DOI: 10.1056/NEJMoa2001316.

Minh BQ, Schmidt HA, Chernomor O, Schrempf D, Woodhams MD, von Haeseler A, Lanfear R. 2020. IQ-TREE 2: New Models and Efficient Methods for Phylogenetic Inference in the Genomic Era. Molecular Biology and Evolution 37:1530-1534. DOI: 10.1093/molbev/msaa015.

Pachetti M, Marini B, Benedetti F, Giudici F, Mauro E, Storici P, Masciovecchio C, Angeletti S, spots include a novel RNA-dependent-RNA polymerase variant. Journal of Translational Medicine 18:179. DOI: 10.1186/s12967-020-02344-6.

Page AJ, Taylor B, Delaney AJ, Soares J, Seemann T, Keane JA, Harris SR. 2016. SNP-sites: rapid efficient extraction of SNPs from multi-FASTA alignments. Microbial Genomics 2:e000056. DOI: 10.1099/mgen.0.000056. 
373 Potdar V, Cherian SS, Deshpande GR, Ullas PT, Yadav PD, Choudhary ML, Gughe R, Vipat V, 374 Jadhav S, Patil S, Nyayanit D, Majumdar T, Walimbe A, Gaikwad S, Dighe H, Shete-

375

376

377

378

379

380

381

382

383

384

385

386

387

388

389

390

391

392

393

394

395
Aich A, Mohandas S, Chowdhury D, Sapkal G, Basu A, Gupta N, Gangakhedkar RR, Giri S, Dar L, Jain A, Malhotra B, Abraham P, Team NIC. 2020. Genomic analysis of SARS-CoV-2 strains among Indians returning from Italy, Iran \& China, \& Italian tourists in India. Indian Journal of Medical Research 151:255. DOI:

10.4103/ijmr.IJMR_1058_20.

Quick J. 2020. nCoV-2019 sequencing protocol. protocols.io. DOI:

dx.doi.org/10.17504/protocols.io.bdp7i5rn.

Rambaut A, Holmes EC, Hill V, O’Toole Á, McCrone JT, Ruis C, Plessis L du, Pybus OG. 2020. A dynamic nomenclature proposal for SARS-CoV-2 to assist genomic epidemiology. bioRxiv:2020.04.17.046086. DOI: 10.1101/2020.04.17.046086.

Seemann T. 2014. Prokka: rapid prokaryotic genome annotation. Bioinformatics (Oxford, England) 30:2068-2069. DOI: 10.1093/bioinformatics/btu153.

Shu Y, McCauley J. 2017. GISAID: Global initiative on sharing all influenza data - from vision to reality. Euro Surveillance: Bulletin Europeen Sur Les Maladies Transmissibles = European Communicable Disease Bulletin 22. DOI: 10.2807/15607917.ES.2017.22.13.30494.

Volz E, Mishra S, Chand M, Barrett JC, Johnson R, Geidelberg L, Hinsley WR, Laydon DJ, Dabrera G, O’Toole Á, Amato R, Ragonnet-Cronin M, Harrison I, Jackson B, Ariani CV, Boyd O, Loman N, McCrone JT, Gonçalves S, Jorgensen D, Myers R, Hill V, Jackson DK, Gaythorpe K, Groves N, Sillitoe J, Kwiatkowski DP, COG-UK, Flaxman S, Ratmann O, Bhatt S, Hopkins S, Gandy A, Rambaut A, Ferguson NM. 2021.

Peer) reviewing PDF | (2020:09:52656:1:1:NEW 22 Jan 2021) 

epidemiological and genetic data. medRxiv:2020.12.30.20249034. DOI:

398 $10.1101 / 2020.12 .30 .20249034$.

399

400

401

402

403

404

405

406

407

408

409

410

Waterhouse AM, Procter JB, Martin DMA, Clamp M, Barton GJ. 2009. Jalview Version 2-a multiple sequence alignment editor and analysis workbench. Bioinformatics 25:11891191. DOI: 10.1093/bioinformatics/btp033.

Yang H-C, Chen C, Wang J-H, Liao H-C, Yang C-T, Chen C-W, Lin Y-C, Kao C-H, Liao JC. 2020. Genomic, geographic and temporal distributions of SARS-CoV-2 mutations. bioRxiv:2020.04.22.055863. DOI: 10.1101/2020.04.22.055863.

Zhou P, Yang X-L, Wang X-G, Hu B, Zhang L, Zhang W, Si H-R, Zhu Y, Li B, Huang C-L, Chen H-D, Chen J, Luo Y, Guo H, Jiang R-D, Liu M-Q, Chen Y, Shen X-R, Wang X, Zheng X-S, Zhao K, Chen Q-J, Deng F, Liu L-L, Yan B, Zhan F-X, Wang Y-Y, Xiao GF, Shi Z-L. 2020. A pneumonia outbreak associated with a new coronavirus of probable bat origin. Nature:1-4. DOI: 10.1038/s41586-020-2012-7. 


\section{Figure 1}

Timeline of symptom onset, illness severity, SARS-CoV-2 RNA sample collection date, hospitalization, and ICU admission of the 11 COVID-19 patients between February and March 2020 in Lebanon.

All patients were admitted to the hospital with varying illness severity. Light blue bars represent asymptomatic patients. Gold bars indicate patients displaying mild symptoms while red bars denote patients with severe cases. Days are numbered sequentially from the $17^{\text {th }}$ of February until the $20^{\text {th }}$ of March. The star symbol indicates the RNA sample collection date while the "*" symbol denotes the date of symptom onset. Orange colored triangles mark the initial hospitalization date. The target symbol indicates admission into the intensive care unit (ICU) and the plus "+" sign is unique to patient S11 where it indicates his admission into the ICU in a different healthcare facility. The forked arrow symbol represents a patient's death.

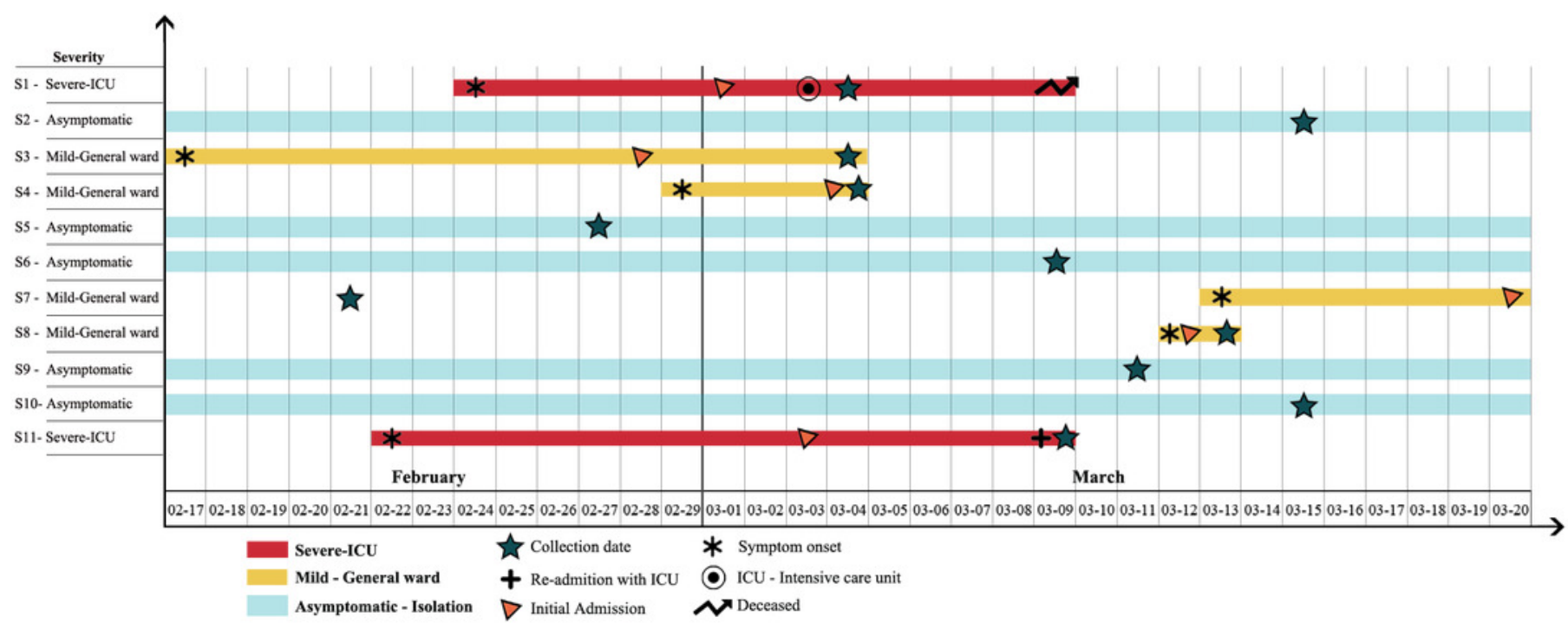




\section{Figure 2}

Figure 2: A maximum-likelihood (ML) phylogenetic tree of SARS-CoV-2 genomic sequences isolated from Lebanon.

Only six genomes, with labels colored red, were selected for the phylogenetic tree to prevent any bias due to $\mathrm{N}$ strings in genomic sequences. Bootstrap values are represented by dark blue circles on the tree branches. Nextstrain clades were defined through different clade colors with light blue denoting the 19A clade, dark blue representing the 20B clade and gold designating the 20A clade. Pangolin lineages are displayed by star symbols (right). Purple stars represent the B.4 lineage, brown denotes the B.1.1 lineage and orange-colored stars stand for the B.1 lineage. Tree scale (upper left corner) connotes for raw branch length and an internal scale system was added for additional stringency. 


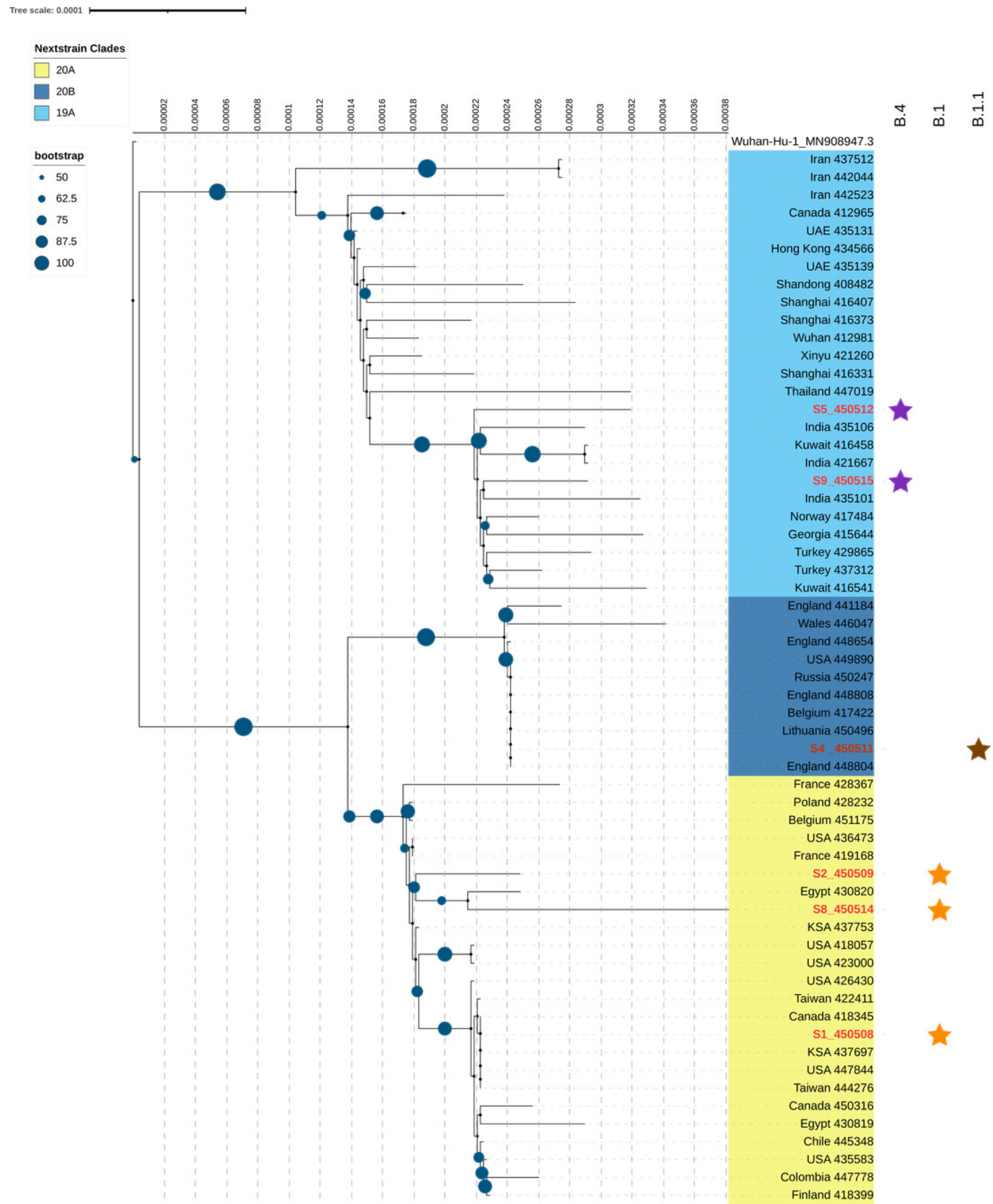




\section{Table $\mathbf{1}$ (on next page)}

Table 1 Clinical characteristics of Lebanese patients with COVID-19 between February and March 2020.

$*$ CT $=$ RT-PCR Cycle Threshold; $\uparrow$ ARDS $=$ Acute respiratory distress syndrome. 


\begin{tabular}{|c|c|c|c|c|c|c|c|c|}
\hline $\begin{array}{c}\text { Accession Number } \\
\text { - GISAID }\end{array}$ & Sample & Gender & $\begin{array}{c}\text { Age } \\
\text { (Years) }\end{array}$ & $\begin{array}{l}\text { Location of } \\
\text { Exposure }\end{array}$ & Sample type & $\mathrm{Ct}^{*}$ & Patient status & Signs and Symptoms \\
\hline EPI_ISL_450508 & S1 & Male & 56 & Egypt & Sputum/PBS & 14.78 & Hospitalized-Deceased & $\begin{array}{c}\text { Early stages: Flu like symptoms } \\
\text { Followed by severe Dyspnea and severe } \\
\text { ARDS. } \dagger \\
\text { Chest X-ray: Patchy bilateral upper lobe } \\
\text { consolidation. }\end{array}$ \\
\hline EPI_ISL_450509 & $\mathrm{S} 2$ & Male & 63 & $\begin{array}{l}\text { Local - } \\
\text { Community } \\
\text { acquired }\end{array}$ & $\begin{array}{c}\text { Nasopharyngeal } \\
\text { VTM }\end{array}$ & 26.15 & Hospitalized-Released & Asymptomatic \\
\hline EPI_ISL_450510 & $\mathrm{S} 3$ & Male & 19 & Iran & $\begin{array}{c}\text { Nasopharyngeal } \\
\text { VTM }\end{array}$ & 37.5 & Hospitalized-Released & Headache, Abdominal pain, and Diarrhea \\
\hline EPI_ISL_450511 & S4 & Female & 33 & $\begin{array}{c}\text { United } \\
\text { Kingdom }\end{array}$ & $\begin{array}{c}\text { Nasopharyngeal } \\
\text { VTM }\end{array}$ & 34.67 & Hospitalized-Released & Rhinorrhea and Headache \\
\hline EPI_ISL_450512 & S5 & Male & 42 & Iran & $\begin{array}{c}\text { Nasopharyngeal } \\
\text { VTM }\end{array}$ & 16.11 & Hospitalized-Released & Asymptomatic \\
\hline EPI_ISL_450513 & S6 & Male & - & France & $\begin{array}{c}\text { Nasopharyngeal } \\
\text { VTM }\end{array}$ & 33.7 & Hospitalized-Released & Asymptomatic \\
\hline EPI_ISL_454420 & S7 & Female & 41 & Iran & $\begin{array}{c}\text { Nasopharyngeal } \\
\text { VTM }\end{array}$ & 20.24 & Hospitalized-Released & Sore Throat and Rhinorrhea \\
\hline EPI_ISL_450514 & S8 & Female & 74 & $\begin{array}{l}\text { Local - } \\
\text { community } \\
\text { acquired }\end{array}$ & $\begin{array}{c}\text { Nasopharyngeal } \\
\text { VTM }\end{array}$ & 33.8 & Hospitalized-Released & Dyspnea \\
\hline EPI_ISL_450515 & S9 & Female & 25 & $\begin{array}{l}\text { United } \\
\text { Kingdom }\end{array}$ & $\begin{array}{c}\text { Nasopharyngeal } \\
\text { VTM }\end{array}$ & 34 & Hospitalized-Released & Asymptomatic \\
\hline EPI_ISL_450516 & S10 & Male & 36 & $\begin{array}{l}\text { Local - } \\
\text { community } \\
\text { acquired }\end{array}$ & Sputum/PBS & - & Hospitalized-Released & Asymptomatic \\
\hline EPI_ISL_450517 & S11 & Male & 22 & Italy & $\begin{array}{c}\text { Nasopharyngeal } \\
\text { VTM }\end{array}$ & - & Hospitalized-Released & $\begin{array}{l}\text { Early stages: Dysuria, Fever and Flu-like } \\
\text { symptoms. } \\
\text { Followed by severe Dyspnea and ARDS. } \\
\text { Chest CT: Bilateral infiltrates with ground } \\
\text { glass appearance. }\end{array}$ \\
\hline
\end{tabular}


$2 * \mathrm{CT}=\mathrm{RT}-\mathrm{PCR}$ Cycle Threshold; $\uparrow \mathrm{ARDS}=$ Acute respiratory distress syndrome. 
Table 2 (on next page)

Table 2: SNP distribution in SARS-CoV-2 genome sequences 
Table 2: SNP distribution in SARS-CoV-2 genome sequences

\begin{tabular}{|c|c|c|c|}
\hline Gene & $\begin{array}{l}\text { High Depth }(\mathrm{DP}>15) \\
\text { nucleotide mutations }\end{array}$ & $\begin{array}{l}\text { Low Depth }(\mathrm{DP}<15) \\
\text { nucleotide mutations }\end{array}$ & Isolates \\
\hline Nsp2 & G1397A & C884T, C1093T, C1884T & S5, S9 \\
\hline Nsp3 & C3037T, A7766G & $\begin{array}{l}\text { C6078T, C6198A, } \\
\text { A6281G, A6282T, } \\
\text { C6285A, C7528T, }\end{array}$ & $\begin{array}{l}\text { S1, S2, S4, S5, S7, S8, S10, } \\
\text { S11 }\end{array}$ \\
\hline Nsp4 & $\mathrm{C} 9118 \mathrm{~T}$ & $\begin{array}{l}\text { G8653T, C8655T, } \\
\text { A8658G, A8897T, } \\
\text { T9860C, T9861G }\end{array}$ & S2, S5, S7, S8, S9, S10, S11 \\
\hline Nsp5 & - & $\mathrm{C} 10074 \mathrm{~T}, \mathrm{~A} 10075 \mathrm{~T}$ & S7, S11 \\
\hline Nsp6 & G11083T & - & S5, S9 \\
\hline Nsp8 & - & A12297T & S10 \\
\hline Nsp10 & $\mathrm{C} 13381 \mathrm{~T}$ & - & $\mathrm{S} 2, \mathrm{~S} 8, \mathrm{~S} 11$ \\
\hline Nsp12 & $\mathrm{C} 14408 \mathrm{~T}$ & $\begin{array}{c}\mathrm{G} 14369 \mathrm{~T}, \mathrm{C} 14703 \mathrm{~T}, \\
\mathrm{C} 14724 \mathrm{~T}, \mathrm{C} 14802 \mathrm{~T}, \\
\mathrm{C} 14993 \mathrm{~T}\end{array}$ & $\mathrm{~S} 1, \mathrm{~S} 2, \mathrm{~S} 4, \mathrm{~S} 7, \mathrm{~S} 8, \mathrm{~S} 10, \mathrm{~S} 11$ \\
\hline Nsp13 & - & G16301T & S7 \\
\hline Nsp14 & $\mathrm{C} 18877 \mathrm{~T}$ & G18670T & $\mathrm{S} 1, \mathrm{~S} 7$ \\
\hline Nsp15 & - & A19499C & S7 \\
\hline $\begin{array}{l}\text { Spike }(\mathrm{S}) \\
\text { gene }\end{array}$ & $\mathrm{A} 23403 \mathrm{G}$ & G22021T, T22092G & $\begin{array}{l}\text { S1, S2, S4, S7, S8, S9, S10, } \\
\text { S11 }\end{array}$ \\
\hline ORF3a & $\begin{array}{c}\mathrm{G} 25563 \mathrm{~T}, \mathrm{C} 25578 \mathrm{~T}, \\
\mathrm{C} 25609 \mathrm{~T}\end{array}$ & $\mathrm{C} 25611 \mathrm{~T}, \mathrm{~A} 25965 \mathrm{G}$ & $\mathrm{S} 1, \mathrm{~S} 2, \mathrm{~S} 5, \mathrm{~S} 8, \mathrm{~S} 9, \mathrm{~S} 10, \mathrm{~S} 11$ \\
\hline ORF7a & - & $\mathrm{C} 27643 \mathrm{~T}$ & $\mathrm{~S} 11$ \\
\hline $\begin{array}{l}\text { None-coding } \\
\text { region }\end{array}$ & - & G27788A, T27789C & S5, S9 \\
\hline N gene & $\begin{array}{l}\text { T28688C, G28881A, } \\
\text { G28882A, G28883C }\end{array}$ & C28354T & S4, S5, S8, S9 \\
\hline $\begin{array}{l}\text { None-coding } \\
\text { region }\end{array}$ & - & G29543T & S7 \\
\hline 3'UTR & G29742T & - & S5, S7, S9 \\
\hline
\end{tabular}




\section{Table $\mathbf{3}$ (on next page)}

Table 3: Novel amino acid mutations in SARS-CoV-2 genomes 
Table 3: Novel amino acid mutations and lineage assignment in SARS-CoV-2 genomes

\begin{tabular}{|c|c|c|c|c|c|c|}
\hline Sample & $\begin{array}{c}\text { Total } \\
\text { number of } \\
\text { SNVs }\end{array}$ & $\begin{array}{l}\text { Genome } \\
\text { Coverage }\end{array}$ & $\begin{array}{c}\text { Novel Amino Acid } \\
\text { changes as of time of } \\
\text { submission }\end{array}$ & $\begin{array}{l}\text { Pangolin Lineage } \\
\text { assignment }\end{array}$ & $\begin{array}{l}\text { SH-alrt } \\
(\%)\end{array}$ & $\begin{array}{c}\text { UFbootstrap } \\
(\%)\end{array}$ \\
\hline S1 & 5 & $>95 \%$ & None & B.1 & 100 & 100 \\
\hline S2 & 6 & $>95 \%$ & None & B.1 & 100 & 100 \\
\hline S3 & $\mathrm{n} / \mathrm{a}$ & $<95 \%$ & $\mathrm{n} / \mathrm{a}$ & B.4 & 100 & 100 \\
\hline S4 & 6 & $>95 \%$ & None & B.1.1 & 100 & 99 \\
\hline S5 & 8 & $>95 \%$ & nsp3: I1683V & B.4 & 100 & 100 \\
\hline S6 & $\mathrm{n} / \mathrm{a}$ & $<95 \%$ & $\mathrm{n} / \mathrm{a}$ & B.1 & 100 & 100 \\
\hline S7 & 16 & $>95 \%$ & $\begin{array}{c}\text { nsp12: C310F; nsp13: } \\
\text { R22I; nsp14: D211Y, } \\
\text { H487P }\end{array}$ & B.1 & 100 & 100 \\
\hline S8 & 10 & $>95 \%$ & $\begin{array}{c}\text { nsp3: S1160Y; nsp4: } \\
\text { M33Ffs }\end{array}$ & B.1 & 100 & 100 \\
\hline S9 & 7 & $>95 \%$ & None & B.4 & 100 & 100 \\
\hline S10 & 18 & $>95 \%$ & $\begin{array}{l}\text { nsp3: N1188V, } \\
\text { T1189N; nsp4: } \\
\text { L436R; nsp8: Q69L; } \\
\text { ORF7b: C12R }\end{array}$ & B.1 & 100 & 100 \\
\hline S11 & 19 & $>95 \%$ & $\begin{array}{c}\text { nsp4: N115Y; nsp12: } \\
\text { S518L; S protein: } \\
\text { M177R }\end{array}$ & B.1 & 100 & 100 \\
\hline
\end{tabular}

